\title{
Sepsis: a frequent topic in a new Editorial Section in INFECTION
}

\author{
J. R. Bogner ${ }^{1}$ \\ Published online: 28 January 2019 \\ (c) Springer-Verlag GmbH Germany, part of Springer Nature 2019
}

Dear Readers of INFECTION. As of January 2019, the term "Sepsis" is found 555 times in our journal. This is the result of a PubMed search which shows that this topic was present also in one of the first issues in the year 1973 [1]. For 45 years, sepsis has been covered with all types of articles, including case reports, original articles and reviews. Latest examples focus on a wide range, from sepsis in neonates over blood stream infection of various types to interesting case reports involving multi-drug-resistant organisms (MDRO) [2-8].

The fact that we frequently publish works on sepsis and related topics as well as the longstanding cooperation with the German Sepsis Society (DSG) led to the consequent decision to form a new Section simply called "Sepsis". We are grateful to the DSG for the long-lasting cooperation and for the opportunity to print the proceedings of the meetings of DSG. We are proud to present Professor Mathias Pletz as the new Section Editor for "Sepsis".

Mathias W. Pletz, Professor for Infectious Diseases, is a board-certified physician for internal medicine, pulmonology and infectious diseases, and the Head of the Institute for Infectious Diseases and Infection Control of the University Hospital in Jena (Germany). He also leads a clinical research group focusing on novel diagnostic and therapeutic strategies against MDRO, funded by the German Ministry for Science and Education.

After his medical training at Leipzig (Houston, TX, USA) and the Basel (Switzerland), he started his residency at the Chest Hospital in Berlin (Germany). Subsequently, he spent 2 years as a postdoctoral researcher at Emory University (Atlanta, GA, USA) working on pneumococci and pneumococcal vaccination. In addition, he served as a guest researcher at the Centers for Disease Control and Prevention (CDC) in Atlanta, exploring the concurrent severe

J. R. Bogner

jbogner@med.lmu.de

Klinik und Poliklinik IV, Sektion Klinische Infektiologie, Klinikum der Universität München, Pettenkoferstr. 8a, 80336 Munich, Germany acute respiratory syndrome (SARS) epidemics. After his return to Germany, he completed his medical training at the Department of Respiratory Medicine at the Hannover Medical School. Among others, he received the Kass Award of the Infectious Diseases Society of America, the Respiratory Infections Award of the European Respiratory Society and the Honor Award of the Centers for Disease Control and Prevention.

Besides the German Sepsis Society, he also serves on the board of directors of the German Competence Network for Community-Acquired Pneumonia (CAPNETZ) and the German Society for Infectious Diseases and the Scientific Advisory Board of the Robert Koch Institute.

We are grateful to Prof. Pletz that he accepted the invitation to become Section Editor at INFECTION.

We encourage our readers to keep on submitting work on sepsis, sepsis markers, outcomes and anti-infective treatment of sepsis. Also the implementation of sepsis bundles, standard operating procedures and measures improving at quality improvement in early detection and diagnosis of sepsis are welcome and continue to have high priority in editorial processing and quick decisions $[9,10]$.

We also would like to thank our reviewers in this field. I really acknowledge every single peer review that is submitted upon our request knowing the hard work invested by the reviewer so often results in a real improvement of articles.

Munich, January 2019

Johannes R. Bogner

Editor-in-Chief INFECTION

\section{Compliance with ethical standards}

Conflict of interest The author declares that he has no conflict of interest. 


\section{References}

1. Austin TW, Wallace JF. Staphylococcus aureus bacteremia: a critical review of its treatment and association with infective endocarditis. Infection. 1973;1:214-7.

2. Jung N, Rieg S. Essentials in the management of S. aureus bloodstream infection. Infection. 2018;46:441-2.

3. Koehler P, Jung N, Kochanek M, Lohneis P, Shimabukuro-Vornhagen A, Boll B 'Lost in Nasal Space': Staphylococcus aureus sepsis associated with Nasal Handkerchief Packing. Infection. 2018. https://doi.org/10.1007/s15010-018-1221-6

4. Giacobbe DR, Del Bono V, Mikulska M, Gustinetti G, Marchese A, Mina F, et al. Impact of a mixed educational and semi-restrictive antimicrobial stewardship project in a large teaching hospital in Northern Italy. Infection. 2017;45:849-56.

5. Rylance J, Nsutebu E, Mergani KO, Grobusch MP, Jacob ST, African Sepsis A. The African Sepsis Alliance: making a difference in the fight against sepsis in Africa. Infection. 2018;46:733-4.

6. Huson MAM, Katete C, Chunda L, Ngoma J, Wallrauch C, Heller $\mathrm{T}$, et al. Application of the qSOFA score to predict mortality in patients with suspected infection in a resource-limited setting in Malawi. Infection. 2017;45:893-6.

7. Assimakopoulos SF, Triantos C, Thomopoulos K, Fligou F, Maroulis I, Marangos M, et al. Gut-origin sepsisin the critically ill patient: pathophysiology and treatment. Infection. 2018;46:751760 https://doi.org/10.1007/s15010-018-1178-5

8. Rolston KV. Polymicrobial pulmonary infections in cancer patients with underlying solid tumors. Infection. 2017;45:245-6.

9. Diab M, Sponholz C, von Loeffelholz C, Scheffel P, Bauer M, Kortgen A, et al. Impact of perioperative liver dysfunction on inhospital mortality and long-term survival in infective endocarditis patients. Infection. 2017;45:857-66.

10. Raupach-Rosin H, Duddeck A, Gehrlich M, Helmke C, Huebner $\mathrm{J}$, Pletz MW, et al. Deficits in knowledge, attitude, and practice towards blood culture sampling: results of a nationwide mixedmethods study among inpatient care physicians in Germany. Infection. 2017;45:433-41. 